\title{
Local Time Variation Of Gravity Wave Momentum Fluxes and Their Relationship with the Tides Derived From LIDAR Measurements
}

\author{
Ryan Agner and Alan Z. Liu \\ Department of Physical Sciences, Embry-Riddle Aeronautical University, Daytona \\ Beach, FL, USA
}

\begin{abstract}
Gravity waves and atmospheric tides have strong interactions in the mesopause region and are major contributors to the large variabilities in this region. How these two large perturbations interact with each other is not well understood. Wind and temperature measurements from a Na LIDAR at the Starfire Optical Range (35.0 N, 106.5 W), New Mexico from 24 nights between 1998 and 2000 are used to derive local time variations of gravity wave momentum flux and corresponding local time tidal variation of the background wind. The diurnal component of the momentum flux shows a clear modulation by the tide. The gravity wave forcing on the diurnal tidal amplitudes is shown to have an altitudinal and directional dependence.
\end{abstract}

Keywords: gravity waves, diurnal tide, LIDAR, momentum flux

\section{Introduction}

Gravity waves in the mesosphere-lower thermosphere (MLT) region have significant effects on the general circulation of the atmosphere, such as creating the cold summer mesopause at the poles (Holton, 1983). These waves also interact with other large scale atmospheric dynamic processes such as the atmospheric thermal tides and planetary waves. The thermal tides are a major feature of the atmosphere including the mesopause region where their amplitudes can reach several tens of meters per second (Zhang and Sheperd, 2005). The main thermal tide reflects the solar heating source with a diurnal (24 hour) period with the semidiurnal (12 hours) also important at certain latitudes (Yuan et al., 2008a). Dissipating gravity waves can affect

Preprint submitted to JournalofAtmosphericandSolar-TerrestrialPhysics October 22, 2015 
the tides, and the tidal wind can in turn affect the propagation and dissipation of gravity waves (Liu et al., 2008). Due to the large variability in gravity wave occurrence and frequency, the effect that these waves have on the larger scale thermal tides is not well understood ( Liu et al., 2013; Ortland and Alexander, 2006).

In most general circulation models (GCMs), the effect of gravity waves are parameterized due to computational constraints. While the zonal mean structure can be simulated reasonably well with a variety of parameterization methods, modeling works have shown notable differences in gravity wave tidal interactions based upon different gravity wave parameterization methods. For example, GCMs which use the Lindzen type (Lindzen, 1981) parameterization (e.g. Whole Atmosphere Community Climate Model) tend to suppress the diurnal tidal amplitude (England et al., 2006; McLandress, 1998). while those that use the Hines Doppler Spread Parameterization (e.g. Canadian Middle Atmosphere Model) tend to increase the diurnal tidal amplitude (McLandress, 1998; Meyer, 1999). In order to avoid the dependence on gravity wave parameterization methods in the study of gravity wave-tide interactions, Watanabe and Miyahara (2009) used the Japanese Atmospheric General circulation model for Upper Atmosphere Research (JAGUAR) to directly simulate both gravity waves and tides. Their results show that gravity waves can either amplify or suppress the tidal wind depending on the season. Even though JAGUAR does not parameterize gravity waves, it still needs parameterizations for the turbulence associated with gravity wave dissipation. The model is also still limited by the resolution, which cannot resolve small scale gravity waves.

Observational analysis can provide insights on the relationships between gravity waves and tides. Due to large differences in their temporal and spatial scales, long-term simultaneous observation of gravity waves and tides are not easy to obtain. There are many case studies of gravity waves interacting with a tidal feature (e.g. Thayaparan et al., 1995; Isler and Fritts, 1996; Nakamura et al., 1997; She et al., 2004; Williams et al., 2006). Due to the highly intermittent and local nature of gravity waves, the results of these studies vary depending on the property of waves and the local tidal perturbation. It is not clear which features occur more frequently and which features are special. Recently, Liu et al. (2014), used a two dimensional numerical model to show that the local time nonlinear interaction between gravity waves and tides are important to the formation of large winds and wind shears in the MLT region. These interactions are shown to be highly dependent on the 
phase of the tide in the region.

In this study, we attempt to address the long-term averaged effects of the gravity wave-tide interactions using LIDAR measurements. Recently, Liu et al. (2013) used individual meteor trail wind detections from a meteor radar over Maui, Hawaii to derive the gravity wave momentum flux and their effects on the diurnal tide over a five-year period. While meteor radar can provide long-term observations, high counts of meteor detections within a short duration ( 1 to 2 hours, to resolve tidal variation) is needed to derive reliable momentum flux (Vincent et al., 2010; Fritts et al., 2012). Na Doppler LIDARs can measure wind at high spatial and temporal resolutions, making it ideal to derive the local time variation of momentum flux. On the other hand, it is very challenging to make long term 24-h continuous observations with a Na LIDAR as has been done by She et al. (2004).

In this work, we use high resolution $\mathrm{Na}$ LIDAR measurement of wind and temperature obtained at Starfire Optical Range (SOR, 35. $\left.0^{\circ} \mathrm{N}, 106.5^{\circ} \mathrm{W}\right)$, New Mexico from 1998 to 2000 to derive local time variations of gravity wave momentum flux and examine their relationship to variations of background wind associated with the diurnal tides. This LIDAR dataset was acquired at night only. Even though there is no continuous coverage, we selected nights when the diurnal tides were strong so the tidal components can be derived reliably. The goal is to examine the average gravity wave-tide relationship. In the following section, the LIDAR data and processing method are described. In section 3 , the results for the composite phase aligned day are presented and the discussion and conclusion is in section 4 .

\section{Data and Method}

The Na LIDAR wind and temperature observations used for this study were obtained at the SOR, located in the Kirtland Air Force Base near Albuquerque, NM. The LIDAR was pointed to five directions at zenith, and $10^{\circ}$ off zenith at North, East, Zenith, South, and West in sequence, to measure line of sight wind, $\mathrm{Na}$ density and temperature in each direction with $90 \mathrm{sec}$ integration time. The raw photon counts were accumulated at $24 \mathrm{~m}$ range resolution and the derived quantities have a range resolution of $480 \mathrm{~m}$. The observations were made at night only, with a total of 51 nights data. Detailed information about the instrument and data can be found in Gardner and Liu (2007). 
For each night, the gravity wave perturbations are deduced by removing a linear trend in time within a two-hour sliding window in each of the five line of sight (LOS) observing directions. This gives gravity wave perturbations with periods less than 2 hours, which include most significant gravity waves in this region (Tang et al., 2002). The linear fit within the 2-hr window in the LOS directions is considered as the LOS background wind. The zonal and meridional components of the background wind are derived from the following equations,

$$
\begin{aligned}
V_{Z} & =w, \\
V_{E} & =u \sin (\theta)+w \cos (\theta), \\
V_{W} & =-u \sin (\theta)+w \cos (\theta), \\
V_{N} & =v \sin (\theta)+w \cos (\theta), \\
V_{S} & =-v \sin (\theta)+w \cos (\theta),
\end{aligned}
$$

where $V_{Z}, V_{E}, V_{W}, V_{N}$ and $V_{S}$ are the LOS background winds from the linear fits, $\theta=10^{\circ}$ is the zenith angle, $u$ and $v$ are the zonal and meridional components of the horizontal background winds respectively, and $w$ is the vertical wind. A similar method for extracting wind perturbations for each night was used by Acott et al. (2011) at Fort Collins, CO. The nightly momentum flux at Fort Collins show similar magnitudes to the momentum flux of the phase aligned composite day derived here.

Since the observations were made at night only, extracting the diurnal variation from the measurements may not be reliable, especially when the diurnal variations are weak or the semidiurnal variations are much stronger. To minimize the uncertainty due to weak tidal activity, only nights which exhibited strong diurnal tidal features were selected for the analysis so that the GW-tide interaction is more clear. The problem of extracting reliable tidal information at a particular altitude from only fractions of a day has been a long standing problem (Crary and Forbes, 1983). Here, we take advantage of the additional vertical structure afforded by the LIDAR to constrain the tidal fits and derive more accurate information about each night. A similar approach was used by Liu et al. (2004). Selection of nights with strong tidal features was performed with a two dimensional sinusoidal least-squares fit in time and altitude on the zonal and meridional background horizontal winds to extract the diurnal and semidiurnal amplitudes and phases. A linear combination of diurnal and semidiurnal sinusoids was used due to the relatively similar amplitudes of each tidal feature at the mid-latitudes ( $L i$ 
and Wang, 2009). An additional exponential growth factor was multiplied with each sinusoid to better represent the change in amplitude with altitude as shown in equation 2 .

$$
\begin{array}{r}
U_{\text {fit }}(z, t)=U_{\text {mean }}+A_{24} * \sin \left(\frac{2 \pi t}{24}+\frac{2 \pi z}{\lambda_{24}}+\phi_{24}\right) * e^{\left(\beta_{24} * z\right)}+\ldots \\
A_{12} * \sin \left(\frac{2 \pi t}{12}+\frac{2 \pi z}{\lambda_{12}}+\phi_{12}\right) * e^{\left(\beta_{12} * z\right)}
\end{array}
$$

where $U_{\text {mean }}$ is the mean, $A$ is the amplitude, $\lambda$ is the vertical wavelength, $\phi$ is the phase $\beta$ is the exponential growth factor, $t$ is the time in hours, $z$ is the altitude and the subscripts 24 and 12 represent the diurnal and semidiurnal components respectively.

The selection of nights for the analysis involved setting limits on several fitted parameters. Nights which exhibited an r-square goodness of fit statistic greater than 0.3 were considered for the analysis. This limit was chosen based upon the balance between the number of nights passing this limit and a visual inspection of the quality of the fit. The diurnal tidal amplitude was required to be greater than $20 \mathrm{~m} / \mathrm{s}$ for the tide to be considered strong. Additionally, a night was required to have more than 10 scan profiles and more than 6 hours of data to be used. Due to differences in the number of profiles in the zonal and meridional directions on some nights, each direction was considered separately for the data binning and analysis.

An example of the two dimensional fit for February 201999 is shown in Figure 1. The wind data from the LIDAR for this night does not show an obvious diurnal or semidiurnal tidal structure but a reconstruction of the tides using the parameters derived from the two dimensional fit represents the observed structure well. The diurnal amplitude for this night is $61 \mathrm{~m} / \mathrm{s} \pm$ $3.5 \mathrm{~m} / \mathrm{s}$ with a vertical wavelength of $22 \mathrm{~km} \pm 0.5 \mathrm{~km}$ while the semidiurnal amplitude is $42 \mathrm{~m} / \mathrm{s} \pm 3.2 \mathrm{~m} / \mathrm{s}$ with a vertical wavelength of $35 \mathrm{~km} \pm 2 \mathrm{~km}$ at the $95 \%$ confidence level.

The selection process resulted in 23 nights in the zonal direction and 19 in the meridional. The distribution of the nights through out the calendar months from 1998 to 2000 for the zonal direction is listed in Table 1 and for the meridional in Table 2. The nights roughly spreads out evenly for all calendar months in both directions, except in July when no observation was available. Only wind data is used in this analysis. 

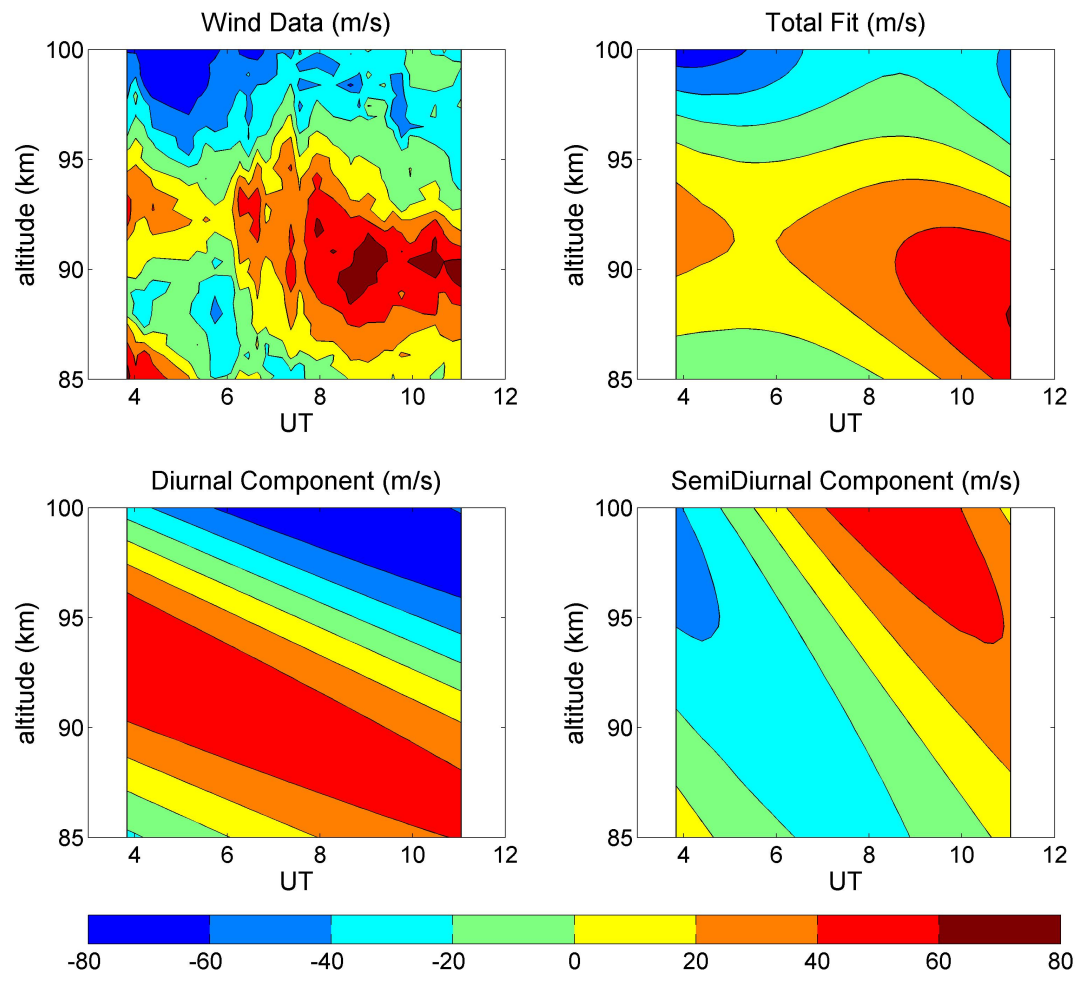

Figure 1: Example of the tidal fits on the zonal wind for the night of October 272000 in $\mathrm{m} / \mathrm{s}$. The top left plot is the raw wind data from the LIDAR, the top right plot is the total fit including both the diurnal and semidiurnal components, the bottom left plot is the diurnal component of the fit, and the bottom left plot is the semidiurnal component of the fit. 
Table 1: Zonal Days exhibiting strong tidal features in each month

\begin{tabular}{|c|c|c|c|c|c|c|c|c|c|c|c|c|}
\hline & Jan & Feb & Mar & Apr & May & Jun & Jul & Aug & Sep & Oct & Nov & Dec \\
\hline 1998 & 0 & 0 & 0 & 0 & 0 & 0 & 0 & 0 & 1 & 0 & 0 & 3 \\
\hline 1999 & 2 & 1 & 0 & 1 & 3 & 0 & 0 & 1 & 0 & 2 & 1 & 1 \\
\hline 2000 & 1 & 0 & 2 & 2 & 1 & 0 & 0 & 0 & 0 & 2 & 0 & 0 \\
\hline
\end{tabular}

Table 2: Meridional Days exhibiting strong tidal features in each month

\begin{tabular}{|c|c|c|c|c|c|c|c|c|c|c|c|c|}
\hline & Jan & Feb & Mar & Apr & May & Jun & Jul & Aug & Sep & Oct & Nov & Dec \\
\hline 1998 & 0 & 0 & 0 & 0 & 0 & 1 & 0 & 0 & 1 & 0 & 0 & 2 \\
\hline 1999 & 1 & 1 & 0 & 0 & 2 & 0 & 0 & 1 & 1 & 1 & 1 & 1 \\
\hline 2000 & 1 & 0 & 2 & 2 & 0 & 0 & 0 & 0 & 0 & 1 & 0 & 0 \\
\hline
\end{tabular}

\subsection{Background Wind, Momentum Flux and Gravity Wave Forcing}

The next step is to calculate the composite momentum flux for a phase aligned composite night. The horizontal wind perturbations at the same tidal phase (not same local time) determined from the fit, were grouped together. This is because the tidal phase has daily and seasonal variabilities ( Lu et al., 2011), and this compositing method gives a proper relationship between gravity wave perturbations and the diurnal tide. We use the tidal phase at $90 \mathrm{~km}$ obtained from the tidal fit for this compositing process. Zonal and meridional wind perturbations are grouped based on diurnal tide phases of zonal and meridional wind, respectively. The vertical flux of horizontal momentum at every phase hour was then calculated with the dual-beam method (Vincent and Reid, 1983)

$$
\begin{aligned}
& \overline{u^{\prime} w^{\prime}}=\frac{\overline{V_{E}^{\prime 2}}-\overline{V_{W}^{\prime 2}}}{4 \sin \theta \cos \theta}, \\
& \overline{v^{\prime} w^{\prime}}=\frac{\overline{V_{N}^{\prime 2}}-\overline{V_{S}^{\prime 2}}}{4 \sin \theta \cos \theta}
\end{aligned}
$$

where the primes denote wave perturbed quantities. The gravity wave forcing on the horizontal wind was calculated as the vertical convergence of 
momentum flux

$$
\begin{aligned}
& U_{a c c}=-\frac{1}{\rho} \frac{d}{d z}\left(\overline{\rho u^{\prime} w^{\prime}}\right)=-\frac{d \overline{u^{\prime} w^{\prime}}}{d z}+\frac{\overline{u^{\prime} w^{\prime}}}{H} \\
& V_{a c c}=-\frac{1}{\rho} \frac{d}{d z}\left(\overline{\rho v^{\prime} w^{\prime}}\right)=-\frac{d \overline{v^{\prime} w^{\prime}}}{d z}+\frac{\overline{v^{\prime} w^{\prime}}}{H}
\end{aligned}
$$

where $H$ is the scale height of the atmospheric density $\rho$ calculated using the average temperature at each altitude and phase hour. The composite diurnal phase aligned background wind, momentum flux and forcing were then fitted with a mean plus 24-hr sinusoidal function at each altitude to obtain their diurnal components.

The effects of gravity wave forcing on the amplitude and phase of the diurnal tide is quantified with the Equivalent Rayleigh Friction (ERF) calculated at each altitude. The ERF was introduced by Forbes et al. (1991) and were used in many studies of gravity wave forcing on the diurnal tides for ground based (Liu et al., 2013), satellite (Lieberman et al., 2010; Xu et al., 2009) and modeling (McLandress, 2002; Chang et al., 2011; Lu et al., 2012) studies. The ERF is defined as

$$
\gamma=-\frac{1}{\hat{u}} \frac{d \hat{u}}{d t}=-\frac{\hat{F}}{\hat{u}} .
$$

where $\hat{u}$ is the complex amplitude of the tide and $d \hat{u} / d t=\hat{F}$ is the complex amplitude of the time tendency due to gravity wave forcing. $\gamma$ is a complex quantity whose real part describes the change in amplitude of the tide and the imaginary part describes the change in phase due to the forcing. To calculate the ERF, the complex amplitudes $\hat{u}=u_{0} e^{-i \phi}$ and $\hat{F}=F_{0} e^{-i \psi}$ are obtained from the composite night fits, which gives the amplitudes $u_{0}$ and $F_{0}$ and phases $\phi$ and $\psi$ of the tidal wind and gravity wave forcing, respectively.

The complex amplitude of the tide in (5) refers to the original tidal amplitude in the absence of gravity wave forcing. From the observation, only the tidal amplitude affected by the gravity waves can be measured. To compensate for this, $\gamma$ was modified as follows (Liu et al., 2013),

$$
\gamma_{\text {mod }}=\frac{\gamma}{1-i \gamma / \omega}
$$

where $\omega=2 \pi / 24 \mathrm{hr}$ is the angular frequency of the diurnal tide. Using this modified form of ERF the change in tidal amplitude due to gravity waves is 

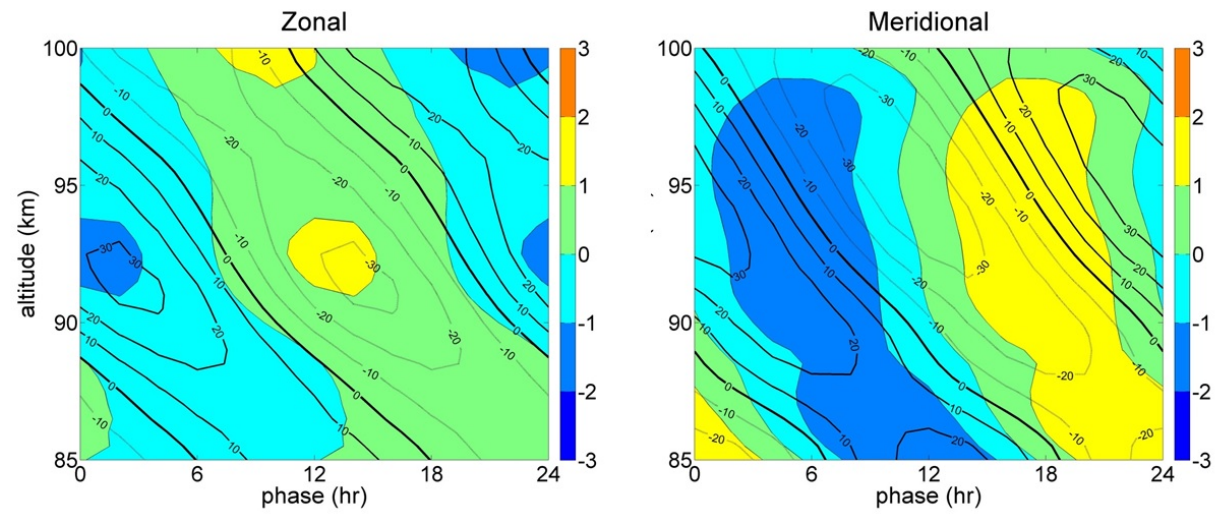

Figure 2: Diurnal variation of the background wind (contour, $\mathrm{m} / \mathrm{s}$ ) and gravity wave momentum flux (color, $\left.\mathrm{m}^{2} / \mathrm{s}^{2}\right)$

then

$$
\frac{d u_{0}}{d t}=-u_{0} \operatorname{Re}\left(\gamma_{\bmod }\right)
$$

and the change in tidal phase is

$$
\frac{d \phi}{d t}=\operatorname{Im}\left(\gamma_{\bmod }\right)
$$

\section{Results}

The diurnal fits at each altitude of the phase aligned composite day momentum flux (colors) and the tidal winds (contours) are shown in Figure 2. The momentum flux contours in both the zonal and meridional directions range from -3 to $3 \mathrm{~m}^{2} / \mathrm{s}^{2}$ with the tidal wind contours from -30 to $30 \mathrm{~m} / \mathrm{s}$. The zonal and meridional momentum fluxes shows a downward tilt below $90 \mathrm{~km}$ matching the tidal wind, indicating some modulation by the diurnal tide. Above $90 \mathrm{~km}$, the diurnal component of the momentum flux is approximately constant in phase while the diurnal tide retains the downward tilt suggesting a weaker tidal modulation of the diurnal component of the momentum flux in the phase aligned composite day.

Figure 3 shows the phase aligned composite day forcing due to gravity waves in both the zonal and meridional directions, again superimposed with the diurnal variation of the background wind. The magnitude of the diurnal component of the zonal forcing ranges from -20 to $20 \mathrm{~m} / \mathrm{s} /$ day and is nearly 

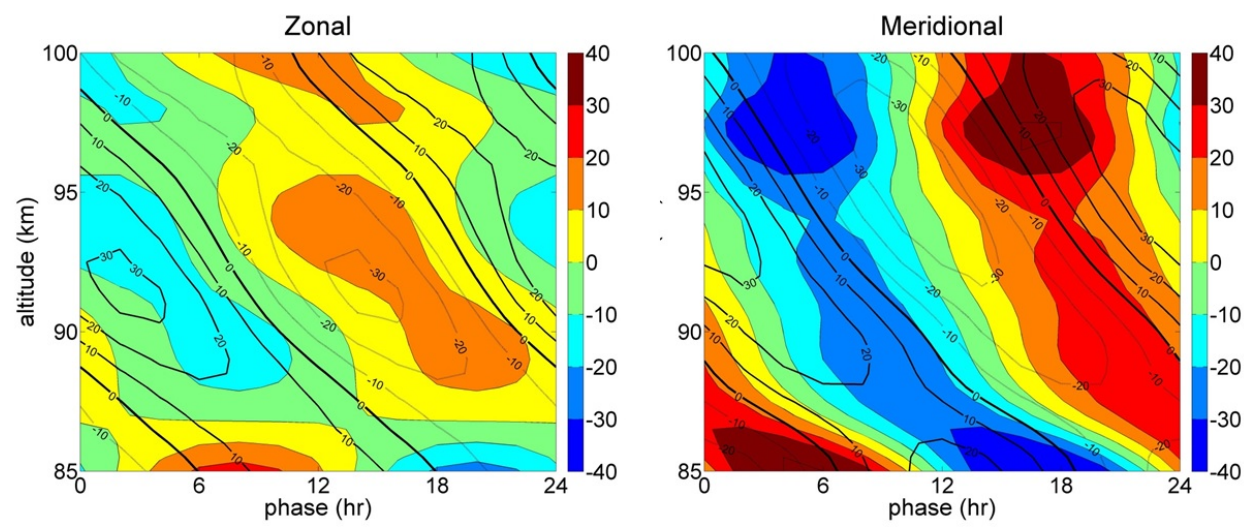

Figure 3: Diurnal variation of the background wind (contour, $\mathrm{m} / \mathrm{s}$ ) and the gravity wave forcing (color, m/s/day)

double that in the meridional direction. The zonal direction shows the forcing to be out of phase between 88 and $98 \mathrm{~km}$ implying that the gravity waves change the amplitude of the diurnal tide at these altitudes with little change in the phase. The partial offsets above and below imply a greater effect on the tidal phase. In the meridional direction, the forcing is out of phase below $90 \mathrm{~km}$ with an approximately $90^{\circ}$ offset above.

The change in phase with altitude for the tidal wind, the momentum flux and the gravity wave forcing is shown in Figure 4 with the left plot showing the zonal phases and the center plot the meridional phases. The error bars are derived from the propagated error for each term. The relative phase difference, defined as the forcing phase minus the tidal phase, is shown in the right plot in Figure 4 for the zonal and meridional directions with the green shaded indicating the areas between $6 \mathrm{hrs}$ and $18 \mathrm{hrs}$ phase difference. The relative phase difference between the zonal tidal wind and the zonal GW forcing at SOR is roughly 12 hours between $88 \mathrm{~km}$ and $97 \mathrm{~km}$ (within the shaded region)and less above and below. In the meridional direction, the relative phase difference between the GW forcing and the tidal wind is of similar magnitude to the zonal direction and is inside the shaded region below $95 \mathrm{~km}$ and outside above.

Figure 5 shows the rates of change of the diurnal tidal amplitude and phase due to gravity waves forcing calculated from the ERF (equations: (7) and (8)) with the associated error bars again derived from propagated errors. The rate of change of the diurnal tide amplitude in the zonal direction has 

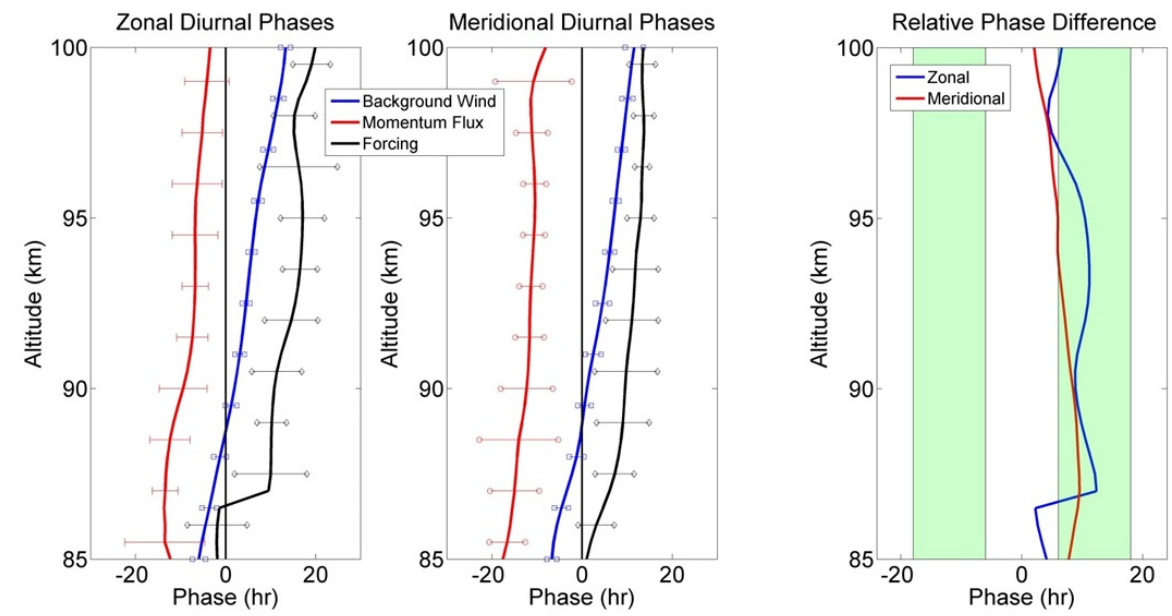

Figure 4: The left two plots are the phase variation with altitude for the background wind (blue), momentum flux (red) and gravity wave forcing (black) in the zonal (left) and meridional (center) directions. The right plot is the phase difference with altitude between the wind and the forcing in the zonal and meridional directions. The shading represents areas where the phase difference is between 6 and 18 hours.

large variations with the forcing acting to damp the tide between $87 \mathrm{~km}$ and $97 \mathrm{~km}$ and enhance it otherwise ranging from -20 to $20 \mathrm{~m} / \mathrm{s} /$ day. In the meridional direction, the GW forcing damps the meridional component of the tide below $93 \mathrm{~km}$ and enhancing it above. The magnitude of the change in meridional tidal amplitude reaches maximums in the upper and lower altitudes that is nearly double that of the zonal direction but the larger error bars make it possible that the magnitude of the amplitude change is similar to the zonal direction.

A remarkably similar altitude dependence of the zonal tide amplitude is reflected in the Watanabe and Miyahara (2009) GCM study which did not use a gravity wave parameterization scheme but instead directly resolved larger scale gravity waves. We are considering only gravity waves with period less than 2 hours suggesting that different scales of gravity waves have similar effects on the diurnal tide.

The gravity wave forcing also acts to delay the zonal and meridional tidal phase at all altitudes, pulling the phase lines down and shortening the local vertical tidal wavelength. The magnitude of the phase change is similar at all altitudes except near $86 \mathrm{~km}$ and $97 \mathrm{~km}$. This is consistent with the Lindzen 


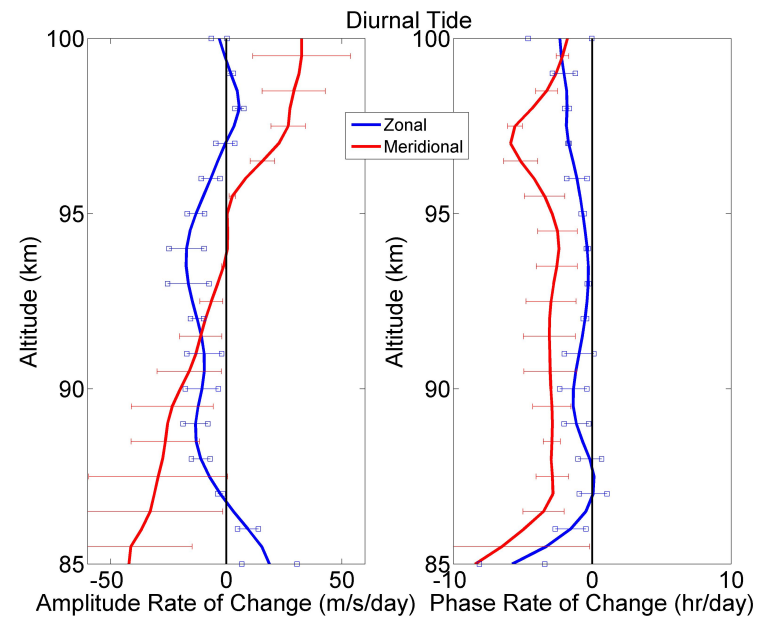

Figure 5: The altitudinal dependence of the rate of change of the diurnal amplitude and phase due to gravity waves

and Hines parameterization schemes but is very different from the Liu et al. (2013) observations which showed an increase in the phase of the diurnal tide at most altitudes. The Watanabe and Miyahara (2009) GCM shows the zonal phase of the tide delayed at all altitudes as consistent with the parameterization schemes and the current observations.

\section{Discussion and Conclusion}

The relationship between high frequency gravity waves and the diurnal tide has been analyzed from two years of Na LIDAR observation at SOR. The diurnal component of the gravity wave momentum flux shows strong modulation by the diurnal tide in the zonal and meridional directions. The amplitude of the diurnal tide is either damped or amplified by gravity waves depending on the altitude. Ortland and Alexander (2006) used a mechanistic tidal model with a gravity wave parameterization scheme to show that the change in diurnal tidal amplitude depends on the phase difference between the gravity wave forcing and the tidal winds. When the gravity wave forcing is more than $90^{\circ}$ out of phase with the tide it will act to increase the tidal amplitude, when the forcing is less than $90^{\circ}$ out of phase with the tide, the tidal amplitude will be decreased. This behavior can be seen in the current results when comparing the relative phase differences with altitude 
in Figure 4 with the tidal amplitude change in Figure 5.

The phase of the zonal diurnal tide at SOR is delayed leading to a local shortening of the vertical tidal wavelength consistent with previous model results. Ortland and Alexander (2006) also showed that the phase of GW forcing always leads that of the tide, resulting in a positive phase difference in agreement with our results.

Using the TIMED satellite, Lieberman et al. (2010) estimated the effects of gravity waves on the migrating diurnal tide in the 80 to $100 \mathrm{~km}$ region by estimating a residual from the momentum equations after subtracting the other large scale terms from the measurements. The residual from the zonal momentum equation was found to lead the zonal wind by $90^{\circ}$ while the residual from the meridional equation was $180^{\circ}$ out of phase. This is similar to our current results with the phase difference in the meridional direction generally greater than the zonal and the gravity wave forcing leading the diurnal wind.

Ortland and Alexander (2006) also showed the gravity wave forcing leading the wind in their model results. The magnitude of the phase difference was highly dependent on the gravity wave source spectrum which can effect the gravity wave breaking altitudes and subsequently, the tidal forcing dependence on altitude. Gravity wave parameterization schemes are typically tuned to match observed zonal mean winds. Each scheme makes different assumptions about gravity wave filtering and breaking processes. Comparisons between observational data and model results with different schemes can help tune the model source spectra to produce tidal amplitudes that are consistent with observations.

The issue of the discrepancy in the gravity wave tidal interactions between parameterization schemes is a complex problem due to the altitudinal and directional dependence of the interactions. Further observational studies from other sites are needed to acquire a more complete understanding . We have also analyzed the gravity wave effects on the semidiurnal tide but no significant relation was found. This could be due to a weaker semidiurnal signature in the available nights of data or larger uncertainties.

\section{Acknowledgments}

The authors would like to thank the reviewers for their work and valuable input in improving this paper. This work is supported by NSF grants AGS1110199 and AGS-1115249. 


\section{References}

Acott, P. E., C.-Y. She, D. A. Krueger, Z. A. Yan, T. Yuan, J. Yue, S. Harrell (2011), Observed nocturnal gravity wave variances and zonal momentum flux in mid-latitude mesopause region over Fort Collins, Colorado, USA, J. Atmos. Sol. -Terr. Phys., 73(4), 449-456.

Chang, L. C., S. E. Palo, and H.-L. Liu (2011), Short-term variability in the migrating diurnal tide caused by interactions with the quasi 2 day wave, J. Geophys. Res., 116, D12112, doi:10.1029/2010JD014996.

Crary, D. J, Forbes, J. M (1983), On the extraction of tidal information from measurements covering a fraction of a day, Geophys. Res. Lett, 10: 580582, doi:10.1029/GL010i007p00580

England, S. L., A. Dobbin, M. J. Harris, N. F. Arnold, and A. D. Aylward (2006), A study into the effects of gravity wave activity on the diurnal tide and airglow emissions in the equatorial mesosphere and lower thermosphere using the Coupled Middle Atmosphere and Thermosphere (CMAT) general circulation model, J. Atmos. Sol. -Terr. Phys., 68, 293, doi:10.1016/j.jastp.2005.05.006.

Forbes, J. M., G. Jun, and M. Saburo (1991), On the interactions between gravity waves and the diurnal propagating tide, Planetary and Space Science, 39(9), 1249-1257.

Fritts, D. C., D. Janches, W. K. Hocking, N. J. Mitchell, and M. J. Taylor (2012), Assessment of gravity wave momentum flux measurement capabilities by meteor radars having different transmitter power and antenna configurations, J. Geophys. Res., 117, D10,108, doi:10.1029/2011JD017174.

Gardner, C. S., and A. Z. Liu (2007), Seasonal variations of the vertical fluxes of heat and horizontal momentum in the mesopause region at Starfire Optical Range, New Mexico, J. Geophys. Res., 112, D09113, doi:10.1029/2005JD006179.

Holton, J.,R., 1983: The influence of gravity wave breaking on the general circulation of the middle atmosphere. J. Atmos. Sci., 40, 24972507.

Isler, J. R., and D. C. Fritts (1996), Gravity wave variability and interaction with lower-frequency motions in the mesosphere and lower thermosphere over hawaii, J. Atmos. Sci., 55(1), 37-48. 
Li, T., C.-Y. She, H.-L. Liu, J. Yue, T. Nakamura, D. A. Krueger, Q. Wu, X. Dou, and S. Wang (2009), Observation of local tidal variability and instability, along with dissipation of diurnal tidal harmonics in the mesopause region over fort collins, colorado (41n, 105w), J. Geophys. Res., 114, D06,106, doi:10.1029/2008JD011089.

Lieberman, R. S., D. A. Ortland, D. M. Riggin, Q. Wu, and C. Jacobi (2010), Momentum budget of the migrating diurnal tide in the mesosphere and lower thermosphere, J. Geophys. Res., 115(D20), D20,105, doi:10.1029/2009JD013684.

Lindzen, R. S. (1981), Turbulence and stress owing to gravity wave and tidal breakdown, J. Geophys. Res., 86, 9707-9714.

Liu, A. Z., R. G. Roble, J. H. Hecht, M. F. Larsen, C. S. Gardner (2004), Unstable layers in the mesopause region observed with $\mathrm{Na}$ lidar during the Turbulent Oxygen Mixing Experiment (TOMEX) campaign, J. Geophys. Res., 109, D02S02, doi:10.1029/2002JD003056.

Liu, A. Z., X. Lu, and S. J. Franke (2013), Diurnal variation of gravity wave momentum flux and its forcing on the diurnal tide, J. Geophys. Res., 118, 1668-1678, doi:10.1029/2012JD018653.

Liu, X., J. Xu, H. -L. Liu, and R. Ma (2008), Nonlinear interactions between gravity waves with different wavelengths and diurnal tide, J. Geophys. Res., 113, D08112, doi:10.1029/2007 JD009136.

Lu, X., A. Z. Liu, J. Oberheide, Q. Wu, T. Li, Z. Li, G. R. Swenson, and S. J. Franke (2011), Seasonal variability of the diurnal tide in the mesosphere and lower thermosphere over Maui, Hawaii (20.7N, 156.3W), J. Geophys. Res., 116(D17), D17,103, doi:10.1029/2011JD015599.

Lu, X., H.-L. Liu, A. Z. Liu, J. Yue, J. M. McInerney, and Z. Li (2012), Momentum budget of the migrating diurnal tide in the Whole Atmosphere Community Climate Model at vernal equinox, J. Geophys. Res., 117(D7), D07,112, 14 PP.

Liu, X., J. Xu, J. Yue, H. L. Liu, and W. Yuan (2014), Large winds and wind shears caused by the nonlinear interactions between gravity waves and tidal backgrounds in the mesosphere and lower thermosphere, J. Geophys. Res. Space Physics, 119, 7698-7708, doi:10.1002/2014JA020221. 
McLandress, C. (1998), On the importance of gravity waves in the middle atmosphere and their parameterization in general circulation models, $J$. Atmos. Sol.-Terr. Phys., 60(14), 1357-1383.

McLandress, C. (2002), The seasonal variation of the propagating diurnal tide in the mesosphere and lower thermosphere. Part II: The role of tidal heating and zonal mean winds, J. Atmos. Sci., 59(5), 907-922.

Meyer, C. K. (1999), Gravity wave interactions with mesospheric planetary waves: A mechanism for penetration into the thermosphereionosphere system, J. Geophys. Res., 104(A12), 28,181-28,196, doi:10.1029/1999JA900346.

Nakamura, T., D. Fritts, J. Isler, T. Tsuda, V. R.A., and I. Reid (1997), Short-period fluctuations of the diurnal tide observed with low-latitude $\mathrm{mf}$ and meteor radars during cadre: Evidence for gravity wave/tidal interactions, J. Geophys. Res., 102(D22), 26,225-26,238, doi:10.1029/96JD03145.

Ortland, D. A., and M. J. Alexander (2006), Gravity wave influence on the global structure of the diurnal tide in the mesosphere and lower thermosphere, J. Geophys. Res., 111, A10S10, doi:10.1029/2005JA011,467.

She, C. Y., T. Li, R. L. Collins, T. Yuan, B. P. Williams, T. Kawahara, J. D. Vance, P. Acott, D. A. Krueger, H.-L. Liu, and M. E. Hagan (2004), Tidal perturbations and variability in the mesopause region over Fort Collins, CO (41N, 105W): Continuous multi-day temperature and wind lidar observations, Geophys. Res. Lett., 31, L24111, doi:10.1029/2004GL021165.

Tang, J., A. Z. Liu, and G. R. Swenson (2002), High frequency gravity waves observed in $\mathrm{OH}$ airglow at Starfire Optical Range, NM: Seasonal variations in momentum flux, Geophys. Res. Lett., 29(20), 1966, doi:10.1029/2002GL015794.

Thayaparan, T., W. K. Hocking, and J. MacDougall (1995), Observational evidence of tidal/gravity wave interactions using the UWO $2 \mathrm{MHz}$ radar, Geophys. Res. Lett., 22(4), 373-376.

Vincent, R. A., and I. M. Reid (1983), HF Doppler measurements of mesospheric gravity wave momentum fluxes, J. Atmos. Sci., 40(5), 1321-1333. 
Vincent, R. A., S. Kovalam, I. M. Reid, and J. P. Younger (2010), Gravity wave flux retrievals using meteor radars, Geophys. Res. Lett., 37(14), L14,802.

Watanabe, S., and S. Miyahara (2009), Quantification of the gravity wave forcing of the migrating diurnal tide in a gravity waveresolving general circulation model, J. Geophys. Res., 114 (D7), D07,110, doi:10.1029/2008JD011218.

Williams, B. P., D. C. Fritts, C. Y. She, and R. A. Goldberg (2006), Gravity wave propagation through a large semidiurnal tide and instabilities in the mesosphere and lower thermosphere during the winter 2003 macwave rocket campaign, Ann. Geophys., 24, 1199-1208.

Xu, J., A. K. Smith, H.-L. Liu, W. Yuan, Q. Wu, G. Jiang, M. G. Mlynczak, and J. M. Russell III (2009), Estimation of the equivalent Rayleigh friction in mesosphere/lower thermosphere region from the migrating diurnal tides observed by TIMED, J. Geophys. Res., 114, D23103, doi:10.1029/2009JD012209.

Yuan T., C. -Y. She, H. Schmidt, D. A. Krueger, S. Reising (2008a) Seasonal variations of semidiurnal tidal-period perturbations in mesopause region temperature, zonal and meridional winds above Fort Collins, CO (40.6N, 105W), J. Geophys. Res., 113, D20103, doi:10.1029/2007JD009687.

Zhang, S. P., and G. G. Shepherd (2005), Variations of the mean winds and diurnal tides in the mesosphere and lower thermosphere observed by WINDII from 1992 to 1996, Geophys. Res. Lett., 32, L14,111, doi:10.1029/2005GL023293. 